\title{
1. Zeolites and MOFs? Dare to Know Them!
}

\author{
Valentin Valtchev and Svetlana Mintova \\ Laboratoire Catalyse et Spectrochimie, Normandie Univ- \\ ENSICAEN-CNRS, 6 Bd Maréchal Juin, 14000 Caen, France.
}

Keywords: Zeolites, MOFs, porous materials, catalysis, separation

\section{Introduction}

A porous material is a solid matter permeable to fluids due to the presence of pores. Behind this short definition are hidden thousands of porous solids with different pore characteristics, chemical natures and structures. Although not visible, the impact of porous solids on our lives is extremely large. It is difficult to conceive modern society without the extensive use of different types of porous solids for industrial and household needs.

The International Union of Pure and Applied Chemistry (IUPAC) divides porous solids into three groups: microporous, with pore size below $2 \mathrm{~nm}$, mesoporous, with pores between 2 and $50 \mathrm{~nm}$, and macroporous, with pores between 50 and $1000 \mathrm{~nm}[1]$. This classification does not reflect the chemical nature and structural ordering of porous solids that have a great impact on their properties. In the present book two families of crystalline porous solids, zeolites and metal-organic frameworks (MOFs) are reviewed. While zeolites are classical microporous materials with pores below $2 \mathrm{~nm}$ and in most cases below $1 \mathrm{~nm}$, some MOF materials exceed the micropore range. However, both classes of materials exhibit crystalline structures and thus pores with well-defined dimensions.

The porous materials first used by mankind were porous carbon and clay-type minerals. However, zeolites were the ones introduced on a large scale with highly technological processes. Among these processes we find fluid catalytic cracking (FCC) and hydrocracking processes, which convert heavy oil fractions to transportation fuels, as well as different separation processes like selective adsorption of aromatic isomers in petrochemistry. Zeolites have been the backbone of the petrochemical industry for over fifty years and their application as heterogeneous catalysts and separation 
materials continue to expand [2,3]. Today, the application of crystalline microporous materials extends far beyond the petrochemical industry, including areas like optics, electronics, medical diagnosis and chemical sensing [4]. New technological challenges, governmental regulations and environmental issues require new materials that can perform better, faster and are environmentally friendly. Hence, the development of new materials in terms of structure types, framework compositions, size and morphology is a continuous task. A steady growth of new zeolite structures is discovered every year, and their number is already 233 [5].

The classical definition of zeolite, which reflects the particularities of zeolite minerals, is "a crystalline porous aluminosilicate built of adjacent $\mathrm{SiO}_{4}$ and $\mathrm{AlO}_{4}$ tetrahedra" [6]. The quest for porous solids with new properties resulted in materials virtually identical to zeolite minerals but with compositions substantially different from the natural counterparts. Starting in the sixties a new family of zeolite materials has been discovered almost every decade. The $\mathrm{Si} / \mathrm{Al}$ ratio, which varies between 1 and 5 in natural zeolites, was extended to synthetic zeolites much richer in silica. The first high silica zeolite, named Beta, was synthesized in the late sixties [7], and in the seventies all-silica zeolite materials were produced [8]. The early eighties were marked by the discovery of aluminophosphate molecular sieves, which were the first silica-free zeolitic materials [9]. Zeolites exhibit well-defined pores, which for industrially relevant zeolites is below $0.8 \mathrm{~nm}$. This confers them shape selectivity, that is, the ability to select which molecules can be adsorbed or reacted inside their pores. However, a relatively small pore diameter is a drawback in the processing of bulky molecules, like those present in heavy oil fractions. The efforts of synthetic chemists to extend the pore dimension of zeolites led to a family of porous materials with pores larger than $2 \mathrm{~nm}$, called ordered mesoporous materials (OMMs) [10]. Ordered mesoporous materials exhibit well-defined channels as their size can be varied between 2 and $50 \mathrm{~nm}[11,12]$. However, the walls building the pore system are amorphous, which is the reason for a low acid strength compared to that of zeolites. Consequently, these materials did not fulfil the great expectations for processing heavy oil fractions.

The latest big family of crystalline porous solids, discovered in the late eighties are metal-organic framework materials (MOFs) [13]. A MOF framework is built of metallic atoms connected by organic linkers. The flexibility available to build such structures is substantially higher than that for crystalline inorganic materials [14]. In just a decade thousands of MOFs were published in open literature. The absence of a strict classification makes the determination of their exact number difficult. 
The objective of this chapter is to introduce these two important families of crystalline porous solids: zeolites, which have been used in the chemical process industry for over fifty years, and MOFs, which are promising for applications that might be different from those typical of zeolite molecular sieves. More detail information of their properties and applications can be found in this book.

\section{Zeolites}

A zeolite is formed under hydrothermal conditions from an alkali-rich aluminosilicate hydrogel system. In such a system, water is a major component playing two roles, a reactant and reaction medium. The alkaline aqueous solutions dissolve and transport material from the polymerized aluminoslilicate gel to growing crystals. The dissolution and transport of aluminosilicate precursors leads to supersaturation and nucleation in certain zones of the system $[2,6]$. Zeolite nucleation is a spontaneous process, which can be controlled to some extent under laboratory conditions. For example, by controlling the number of nuclei in the system, the ultimate crystal size can be tuned (since, after exhausting the nutrient pool, crystal growth stops) [15]. The key parameters controlling zeolite formation are gel composition and crystallization temperature. The previous history of the reactants, their purity and their mixing may also influence the zeolite synthesis. The aging time of the precursor gel is another important parameter that appears critical for some types of zeolites. The crystallization temperatures vary between 80 and $180^{\circ} \mathrm{C}$, but syntheses at ambient conditions or at temperature much higher than $180^{\circ} \mathrm{C}$ are also reported [6]. The crystallization of different types of zeolites may last from several minutes to several weeks. The crystallization time is important not only for obtaining a highly crystalline zeolite material but also to avoid the transformation of a desired product in another phase. Zeolites are metastable phases that may transform into more stable and denser crystals upon long contact with the mother liquor.

The general chemical composition of a zeolite is $\mathrm{Me}_{2 / n} \mathrm{O}: \mathrm{Al}_{2} \mathrm{O}_{3}: x \mathrm{SiO}_{2}$ : $y \mathrm{H}_{2} \mathrm{O}$, where Me is the metal cation (usually alkali or alkaline earth), $n$ is the charge of the cation, and $x$ and $y$ depend on the zeolite type. The framework silicon/aluminum ratio $(\mathrm{Si} / \mathrm{Al})$ is always greater than 1 since Löwenstein's rule prohibits two neighboring aluminum tetrahedra, i.e. Al-O-Al bonds are forbidden due to unfavourable electrostatic interactions [16]. 


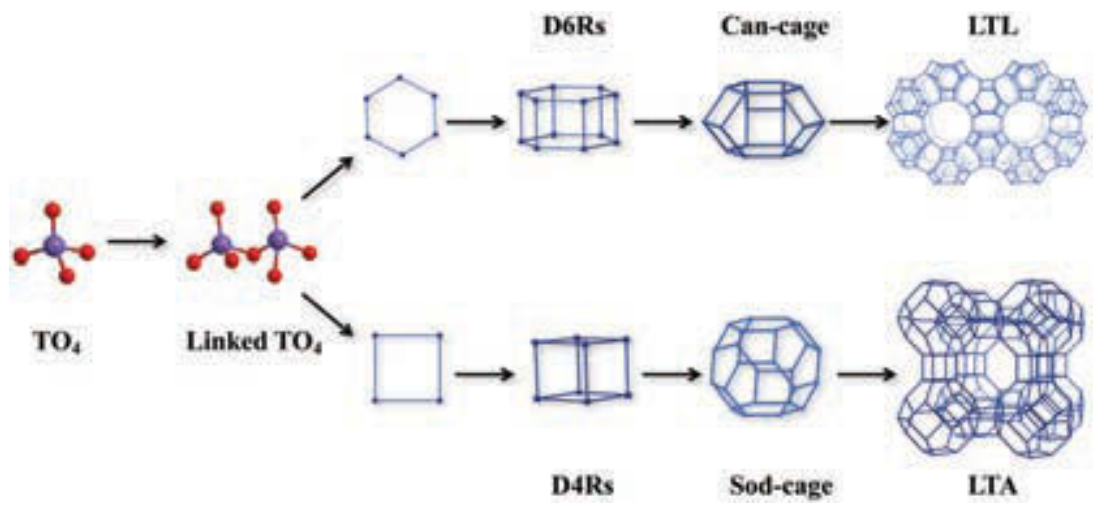

Figure 1. Schematic representation of the formation of zeolite building units and the resultant zeolite structures.

The network of pores and cavities in the zeolite framework is structured by templating species, which can be removed after the zeolite formation. In natural zeolites, the role of the template is played by alkali metal-water clusters. Barrer and Denny firstly introduced tetraalkylammonium cations in zeolite synthesis, which resulted in the formation of silica- richer zeolites [17]. This approach was further extended to the synthesis of very high silica and all-silica zeolite structures [18]. Besides extending the Si/Al ratio to infinity, the use of organic templates provided more flexibility for the incorporation of transition metals in zeolite frameworks. Zeolites with certain amounts of framework Ti, B, P, Fe, Ga or Ge were obtained, and some of them, like Ti-silicate-1 (TS-1), attained great commercial success [19]. It is worth noting the impact of $\mathrm{Ge}$ on the synthesis of extra-large pore zeolites. Owing to the smaller angle of Si-O-Ge and Ge-O-Ge compared to Si-O-Si bonds, Ge-rich initial systems form small (four- and three-membered) ring units and thus zeolites with lower framework density. Some of these materials exhibited pores with size between 1 and $2 \mathrm{~nm}$ and bridged the gap between classical zeolites and mesoporous materials [20]. Unfortunately, none of these materials reached the stage of industrial use due to cost and thermal stability issues.

Zeolites are often conceptually classified by the units that compose the zeolite structure [5]. The so-called "secondary building units" (SBUs) are simple polyhedra that, arranged in different ways by symmetrical operations, result in the different framework types (Figure 1).

The number of T-atoms (that is, the atoms in "tetrahedral" coordination, namely $\mathrm{Si}$ and $\mathrm{Al}$ ) forming a zeolite channel determine to a great extent the size of the pore opening, which is an important characteristic of 
microporous molecular sieves. Consequently, zeolites are classified according to the number of T-atoms building the ring (n-MR):

- small pore zeolites: 8-MR, with diameters $c a .4 \AA$

- medium pore zeolites: $10-\mathrm{MR}$, with diameters $c a .5 .5 \AA$

- large pore zeolites: 12-MR, with diameters $c a .7 .5 \AA$

- extra-large pore zeolites: >14-MR, with diameters over $8 \AA$

The replacement of $\mathrm{Si}$ by $\mathrm{Al}$ in a zeolite structure introduces a negative charge on the framework, which is compensated by cations situated in the channels. These charge-balancing cations are exchangeable and extensively used to modify the properties of zeolites. The replacement of an alkali cation by proton $\left(\mathrm{H}^{+}\right)$converts a zeolite into a solid acid, which is largely exploited for preparation of heterogeneous catalysts. Ion exchange properties of zeolites are also intensively used for solving environmental issues, such as the capture of $\mathrm{Cs}^{+}, \mathrm{Sr}^{2+}$ or $\mathrm{NH}_{4}^{+}$, or the purification or softening of water for domestic, agriculture and industrial necessities.

Physicochemical properties of zeolites are addressed in a number of books and specialized reviews. The most important properties that make zeolites exceptional heterogeneous catalysts, molecular sieves and ion exchangers are mentioned next:

- Well-defined crystalline porous structure with variable dimensions, connectivity and geometry of the pores [21]. The pore system of zeolites is the basis of shape selectivity in catalytic and separation processes. The zeolite channel system is able to separate molecules with differences smaller than 1 Å. The pore system of zeolites also determines:

- The micropore volume (up to $0.35 \mathrm{~cm}^{3} \mathrm{~g}^{-1}$ ).

- The specific surface area (up to $850 \mathrm{~m}^{2} \mathrm{~g}^{-1}$ ).

- Variable chemical composition. Zeolite chemistry can be tuned in very wide ranges, first by varying the $\mathrm{Si} / \mathrm{Al}$ ratio from 1 to infinity and second by the incorporation of heteroatoms into the zeolite framework. The ion exchange properties of zeolites offer an additional level of flexibility in tuning zeolite chemistry. Important properties related to the chemical composition of zeolites are:

- Chemical and thermal stability

- Hydrophilic/hydrophobic interactions

- Number, strength and distribution of Brønsted and Lewis acid sites

- Environmentally friendly materials. 
- Acid/base properties of zeolites combined with exceptional shape selectivity, thermal and mechanical stability make zeolites ideal heterogeneous catalysts [22]. The catalytic application of zeolites is demonstrated in several chapters in this book. Shape selectivity and dipolar interactions between guest species and zeolite structures are the basis of the wide use of zeolite as adsorption and separation materials. These applications strongly depend on the pore characteristics, the nature of the charge-balancing cations and on the hydrophobic/hydrophilic character of the zeolite framework [23]. It is worth noting that zeolite applications are not limited to large-scale chemical processes. Recently zeolitic materials have also been used in electronic, optical and medical applications [24].

\section{Metal-organic Frameworks}

Metal-organic frameworks are hybrid crystalline porous materials with frameworks consisting of inorganic building units (metal ions or clusters) and organic linkers connected by coordination bonds of moderate strength (Figure 2) [25]. The metal ions employed in the synthesis of MOFs could be mono $(\mathrm{Cu})$, bi $(\mathrm{Zn}, \mathrm{Mn}, \mathrm{Co}, \mathrm{Cu})$, tri $(\mathrm{Cr}, \mathrm{Fe}, \mathrm{Al})$ and tetravalent $(\mathrm{Zr})$. More complex clusters such as $\mathrm{Cr}_{3} \mathrm{O}\left(\mathrm{H}_{2} \mathrm{O}\right)_{3}(\mathrm{COO})_{6}, \mathrm{Zr}_{6} \mathrm{O}_{4}(\mathrm{OH})_{10}\left(\mathrm{H}_{2} \mathrm{O}\right)_{6}$ $(\mathrm{COO})_{6}, \mathrm{Zn}_{4} \mathrm{O}(\mathrm{COO})_{6}$ or $\mathrm{Cu}_{2}(\mathrm{COO})_{4}$ are also used [26]. There are different approaches for the synthesis of stable MOFs, including modulated synthesis, isoreticular expansion and topology-guided design [27]. The

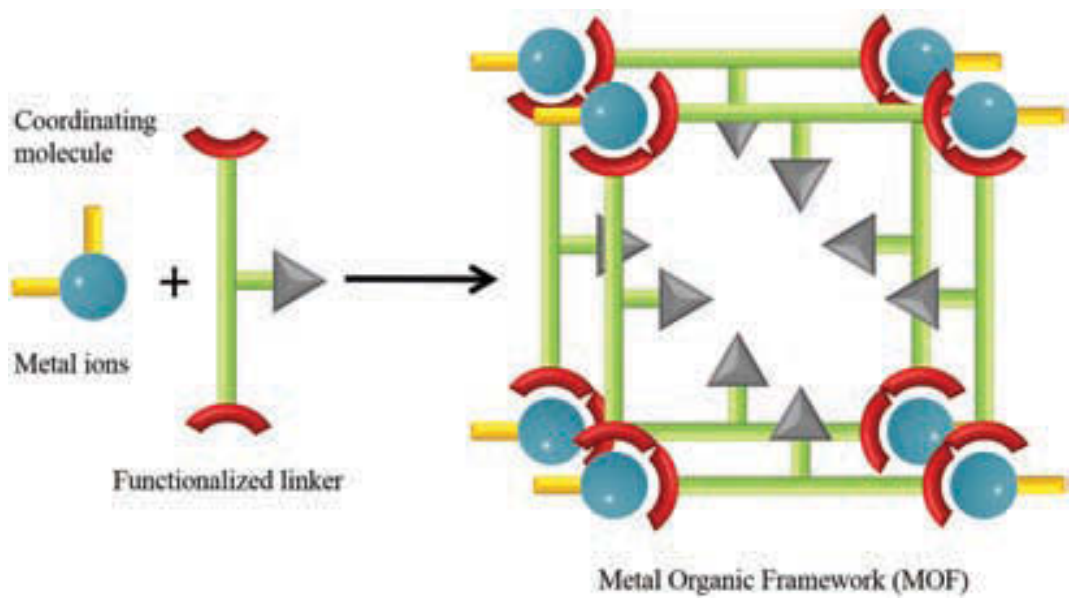

Figure 2. Schematic representation of the formation of a MOF structure. 
presence of diverse inorganic and organic components in MOFs provides a large number of structures with various properties, which will be described briefly following the sequence followed above for zeolites.

The crystalline nature of MOF materials permits a precise structural determination by classical experimental methods such as XRD, TEM and spectroscopic techniques such as NMR, UV-vis and Raman. In addition, computational chemistry is widely applied to predict new materials [28]. In general, MOFs exhibit much lower framework density than zeolites, which can be as low as $0.13 \mathrm{~g} \mathrm{~cm}^{-3}$. Consequently, some MOF materials exhibit $90 \%$ free volume and specific surface areas up to $7000 \mathrm{~m}^{2} \mathrm{~g}^{-1}$.

The stability of any crystalline material is defined as the resistance of the structure to degradation upon heating, cooling or exposure to chemically aggressive environments. The stability of MOFs is governed by multiple factors, including $\mathrm{pK}_{\mathrm{a}}$ of ligands, oxidation state, reduction potential and ionic radius of the metal ions, metal-ligand coordination geometry, and hydrophobicity of the structure [29]. The chemical weak spot of MOFs is usually the metal-linker bond that hydrolyses in aqueous medium, yielding a protonated linker and a de-ligated inorganic moiety. Many examples have demonstrated that both acidic and basic solutions accelerate the dissolution of MOF structures.

Although the general perception is that the stability of MOFs is limited, there are examples of fairly stable structures. For instance zeolitic imidazolate frameworks (ZIFs), metal-azolate (MAF, ZIF-57), and 'zeolitelike MOFs' (ZMOFs) combining azolate and carboxylate coordination capabilities on a single linker show substantially improved chemical and hydrothermal stability [30-32]. ZIF-8 is one of the materials exhibiting a high hydrothermal stability due to the fact that its pore apertures lack polar groups and, thus, the material is hydrophobic. MIL-type MOFs are based on trivalent metals $(\mathrm{Cr}, \mathrm{Al}, \mathrm{Fe})$ or lanthanides $(\mathrm{Eu}, \mathrm{Tb}, \mathrm{Y})$, which form strong bonds with oxygen-anion-terminated linkers and exhibit high chemical stability [33,34]. The hydrothermal stability of various MOFs under steaming at different pressures and temperatures was studied. The most stable under hydrothermal conditions MOFs are zirconium-based UiO-66, MIL-14 and hydrophobic ZIFs [35]. The mechanical stability of MOFs has been found to depend on the valence of the inorganic component and on there structural features. For example, MOFs based on divalent metals have higher mechanical stability as a result of the high strength of their inorganic-linker bond and are therefore more resistant to crystal deformation and damage [36]. 
MOF materials have a number of exceptional properties that make them very promising for future applications in fields where high specific surface area and pore volume are required. In addition, an exceptionally high pore volume and specific surface area combined with a diverse surface chemistry, might extend the application of porous materials beyond that of zeolites. At present, however, the applications considered for MOFs are mainly conceptual because their performance has only been assessed under ideal conditions and collected data is not sufficient to ensure an economically successful application.

MOFs exhibit isolated, well-defined active sites including open metal sites, metalloporphyrins and reactive functional (inorganic or organic) groups, which can serve different types of reactions. Consequently, MOFs have been intensively studied as heterogeneous catalysts. Although there has been no breakthrough resulting from MOF catalysis so far, a number of examples demonstrate the potential of these materials. The vanadium-based MOFs (MIL-47, MOF-48) have been shown to be stable, catalytically active and highly selective in the conversion of $\mathrm{CH}_{4}$ and $\mathrm{CO}$ to acetic acid [37]. Another example is the $\mathrm{Ce}^{4+}$-driven water oxidation reactions catalysed by MOF [38]. Gas-phase catalysis with MOFs materials has been studied as well [39]. An important prospective of MOFs was recently reviewed, in which several single-site catalytic functions can be combined within the framework and used as powerful enzyme-mimicking materials [40].

Certain MOF materials are exceptional adsorbents in terms of the available pore volume [41]. Water-stable MOFs could possibly be applied to effectively uptake gases under moist conditions. There are an increasing number of studies on the use of stable MOFs for wastewater remediation. Targeted compounds in water systems include $\mathrm{SO}_{\mathrm{x}}, \mathrm{NO}_{\mathrm{x}}$, greenhouse gases, VOCs, dyes, drugs, pharmaceuticals, organic chemicals, metal ions, etc. Experimental results have revealed that MOFs have been efficient in adsorbing toxic gases under dry conditions but their adsorption ability was reduced in a humid atmosphere due to the competitive adsorption of water. One exception is $\mathrm{NH}_{3}$, where the decrease in adsorption capacity was negligible, suggesting that ammonia could be removed by a MOF sorbent under both dry and humid conditions. MOFs have also been extensively studied for the past decade as promising hydrogen storage materials [42]. However, many technical problems still remain to be encountered in order to reach practical application.

Water-stable MOFs are extensively employed in membrane separation applications, such as pervaporation, steam separation, desalination, 
and wastewater treatment. Whether MOFs can fully maintain their functions and structure across multi-cycle applications remains a questionable challenge.

Potential applications of MOFs as chemical sensors and adsorbents for the detection and removal of heavy-metal ions have been envisaged [43]. The wide variety of organic linkers and metal nodes that can be incorporated into MOFs could be selected in a way to increase the sensitivity and selectivity towards particular analytes. MOFs could be used as a functional sensing element or as an auxiliary filtering element, while the real-time gas sensing response could be monitored through various methods. For example, electrical property-based sensors were used to monitor the adsorption of analytes within the pores by measuring changes in the conductivity, impedance or resistance of the sensing material. The potential of MOFs in this field is fairly high since the stability of the materials is not a primary concern.

MOFs have also been targeted for biological applications mainly for the controlled delivery of active ingredients. The requirements for MOFs in this field are not only relevant functionalities but also good biocompatibility and degradation under physiological conditions, as they might lead to potential toxicity issues. Considering that a large number of therapeutic molecules possess polycomplex groups in their structure, there are continuous efforts to use active ingredients for the construction of bio-MOFs [44]. Although significant progress has been made in the preparation of bio-MOFs, the possible bio-related application is still in its infancy.

\section{Summary and outlook}

Zeolites and metal-organic frameworks, two important families of crystalline molecular sieve materials, were reviewed, introducing information on the preparation, physicochemical properties and applications that are expanded on later in this book. The two families exhibit a multitude of similarities, like crystalline structure and well-defined pore system, surface functionality and chemical reactivity. On the other hand, the two families differ substantially in terms of chemical nature, pore size and available pore volume, thermal and chemical stability.

Zeolites are inorganic materials with robust frameworks that withstand elevated temperatures and harsh chemical environments. Owing to their high stability, strong acid/base properties and exceptional selectivity, aluminosilicate zeolites are the most successful catalysts in the chemical 
process industry. Zeolite molecular sieves have been actively used more than half a century and new processes based on these exceptional materials continue to be reported. On the other hand, the pore size, volume, and specific surface area of zeolites are substantially lower than that of MOF molecular sieves. The latter exhibit much higher available pore volume and often much larger (meso)pores. The surface chemistry of MOFs can be modulated by in situ or post-synthesis methods and thus adapted to different applications. In the last two decades, MOFs have been the most actively studied family of porous materials and great advances in their preparation, modification and stability have been achieved. It seems, however, that MOF molecular sieves cannot afford the industrial requirements of stability, long lifetime and moderate production cost, yet. Thus, zeolites remain unbeatable as heterogeneous catalysts, adsorbents and ion exchangers. Nevertheless, MOF materials will certainly be used in the future, most probably in areas where they don't compete with zeolites in terms of stability and production price. The fact that these promising porous materials are still not industrially employed may lie in the traditional thinking of applied chemists, which is always biased towards applications where zeolites already have commercial success. We believe that it will not be long until the first industrial use of a MOF material is reported.

\section{Acknowledgements}

The authors acknowledge the financial support from ANR project 15-CEo6ooo4.

\section{References}

[1] Thommes, M., Kaneko, K., Neimark, A.V., Olivier, J.P., Rodriguez-Reinoso, F., Rouquerol, J., Sing, K.S.W. Pure Appl. Chem. 2015, 87, 1051.

[2] Breck, D. Zeolite Molecular Sieves, John Wiley \& Sons, 1974.

[3] Martínez, C., Corma, A. Coord. Chem. Rev. 2011, 255, $155^{8}$.

[4] Mintova, S., Jaber, M., Valtchev, V. Chem. Soc. Rev. 2015, 44, 7207.

[5] http://www.iza-structure.org/databases/

[6] Barrer, R.M. Hydrothermal Chemistry of Zeolites, Academic Press, 1982.

[7] Wadlinger, R.L., Kerr, G.T., Rossinski, E.J. US Patent 3,308,o69, 1967.

[8] Flanigen, E.M., Bennet, J.M., Grose, R.W., Cohen, J.P., Kirchner, R.L., Smith, J.V. Nature 1978, 271,512 .

[9] Wilson, S.T., Lok, B.M., Messina, C.A., Cannan, T.R., Flanigen, E.M.J. Am. Chem. Soc. 1982, $104,1146$. 
[10] Kresge, C.T., Leonowicz, M.E., Roth, W.J., Vartuli, J.C., Beck, J.S. Nature 1992, 359, 710.

[11] Beck, J.S., Vartuli, J.C., Roth, W.J., Leonowicz, M.E., Kresge, C.T., Schmitt, K.D., Chu, C.T.W., Olson, D.H., Sheppard, E.W., McCullen, S.B., Higgins, J.B., Schlenker, J.L. J. Am. Chem. Soc. 1992, 114, 10834.

[12] Zhao, D., Feng, J., Huo, Q., Melosh, N., Frederickson, G.H., Chmelka, B.F., Stucky, G.D. Science 1998, 279, 548.

[13] Li, H., Eddaoudi, M., O'Keeffe, M., Yaghi, O.M. Nature 1999, 402, 276.

[14] Chae, H.K., Siberio-Perez, D.Y., Kim, J., Go, Y., Eddaoudi, M., Matzger, A.J., O'Keeffe, M., Yaghi, O.M. Nature 2004, 427, 276.

[15] Tosheva, L., Valtchev, V. Chem. Mater. 2005, 17, 2494.

[16] Loewenstein, W. Am. Mineral. 1954, 39, 92.

[17] Barrer, R.M., Denny, P.J.J. Chem. Soc. 1961, 983.

[18] Lobo, R.F., Zones, S.I., Davis, M.E.J. Incl. Phenom. Mol. Recogn. Chem. 1995, 21, 47.

[19] Bellussi, G., Carati, A., Clerici, M.G., Esposito, A. Stud. Surf. Sci. Catal. 1991, 63, 421.

[20] Jiang, J., Yu, J., Corma, A. Angew. Chem. Int. Ed. 2010, 49, 3120.

[21] Dyer, A. An introduction to zeolite molecular sieves, John Wiley \& Sons, 1988.

[22] Weitkamp, J., Puppe, L. Catalysis and Zeolites: Fundamentals and Applications, Springer, 1999 .

[23] Di Renzo, F., Fajula, F. Stud. Surf. Sci. Catal. 2005, 157, 1-12.

[24] Sherman, J.D. PNAS 1999, 96, 3471.

[25] O'Keeffe, M., Yaghi, O.M. Chem. Rev. 2012, 112, 675 .

[26] Stock, N., Biswas, S. Chem. Rev. 2012, 112, 933.

[27] Bai, Y., Dou, Y., Xie, L.H., Rutledge, W., Li, J.R., Zhou, H.C. Chem. Soc. Rev. 2016, 45, 2327.

[28] Colon, Y.J., Snurr, R.Q. Chem. Soc. Rev. 2014, 43, 5735.

[29] Chapman, K.W., Halder, G.J., Chupas, P.J.J. Am. Chem. Soc. 2009, 131, 17546.

[3o] Eddaoudi, M., Sava, D.F., Eubank, J.F., Adil, K., Guillerm, V. Chem. Soc. Rev. 2015, 44, 228.

[31] Zhang, J.P., Zhang, Y.B., Lin, J.B., Chen, X.M. Chem. Rev. 2012, 112, 1001.

[32] Liu, Y., Kravtsov, V.C., Eddaoudi, M. Angew. Chem. Int. Ed. 2008, 47, 8446.

[33] Férey, G., Mellot-Draznieks, C., Serre, C., Millange, F., Dutour, J., Surblé, S., Margiolaki, I. Science 2005, 309, 2040.

[34] Alezi, D., Peedikakkal, A.M.P., Weseliński, L.J., Wojtas, R., Eddaoudi M. J. Am. Chem. Soc. $2015,137,5421$.

[35] Nguyen, N.T.T., Furukawa, H., Gándara, F., Nguyen, H.T., Cordova, K.E., Yaghi, O.M. Angew. Chem. Int. Ed. 2014, 53, 10645 .

[36] Wu, H., Yildirim, T., Zhou, W.J. Phys. Chem. Lett. 2013, 4, 925.

[37] Phan, A., Czaja, A.U., Gándara, F., Knobler, C.B., Yaghi, O. Inorg. Chem. 2011, 50, 7388.

[38] Wang, C., Wang, J.L., Lin, W.J. Am. Chem. Soc. 2012, 134, 19895.

[39] Nguyen, H.G.T., Schweitzer, N.M., Chang, C.Y., Drake, T.L., So, M.C., Stair, P.C., Farha, O.K., Hupp, J.T., Nguyen, S.B.T. ACS Catal. 2014, 4, 2496.

[40] Rogge, S.M.J., Bavykina, A., Hajek, J., Garcia, H., Olivos-Suarez, A.I., Sepúlveda-Escribano, A., Vimont, A., Clet, G., Bazin, P., Kapteijn, F., Daturi, M., Ramos-Fernandez, E.V., Llabrés i Xamena, F.X., Van Speybroeck, V., Gascon, J. Chem. Soc. Rev. 2017, 46, 3134.

[41] Wang, C., Liu, X., Demir, N.K., Chenb, J.P., Li, K. Chem. Soc. Rev. 2016, 45, 5107.

[42] Suh, M.P., Park, H.J., Prasad, T.K., Lim, D.W. Chem. Rev. 2012, 112, 782.

[43] Wales, D.J., Grand, J., Ting, V.P., Burke, R.D., Edler, K.J., Bowen, C.R, Mintova, S., Burrow, A.D. Chem. Soc. Rev. 2015, 44, 4290.

[44] Gimenez-Marques, M., Hidalgo, T., Serre, C., Horcajada, P. Coord. Chem. Rev. 2015, 307, 342. 
